\title{
Human chorionic gonadotropin $\beta$ subunit affects the expression of apoptosis-regulating factors in ovarian cancer
}

\author{
ANNA SZCZERBA ${ }^{1 *}$, ALEKSANDRA ŚLIWA $^{1 *}$, MARTA KUBICZAK $^{1}$, \\ EWA NOWAK-MARKWITZ ${ }^{2}$ and ANNA JANKOWSKA ${ }^{1}$
}

\begin{abstract}
Departments of ${ }^{1}$ Cell Biology and ${ }^{2}$ Gynecologic Oncology, Poznan University of Medical Sciences, 60-806 Poznan, Poland
\end{abstract}
Received April 16, 2015; Accepted September 22, 2015

DOI: $10.3892 / o r .2015 .4386$

\begin{abstract}
Expression of human chorionic gonadotropin, especially its free $\beta$ subunit (hCG $\beta$ ) were shown to play an important role in cancer growth, invasion and metastasis. It is postulated that hCG $\beta$ is one of the factors determining cancer cell survival. To test this hypothesis, we applied two models: an in vitro model of ovarian cancer using OVCAR-3 and SKOV-3 cell lines transfected with the $C G B 5$ gene and an in vivo model of ovarian cancer tissues. The material was tested against changes in expression level of genes encoding factors involved in apoptosis: $B C L 2, B A X$ and BIRC5. Overexpression of hCG $\beta$ was found to cause a decrease in expression of the analyzed genes in the transfected cells compared with the control cells. In ovarian cancer tissues, high expression of $C G B$ was related to significantly lower $B C L 2$ but higher $B A X$ and BIRC5 transcript levels. Moreover, a low $B C L 2 / B A X$ ratio, characteristic of advanced stages of ovarian cancer, was revealed. Since tumors were discriminated by a significantly lower $L H C G R$ level than the level noted in healthy fallopian tubes and ovaries, it may be stated that the effect of hCG $\beta$ on changes in the expression of apoptosis-regulating agents observed in ovarian cancer is LHCGR-independent. The results of the study suggest that the biological effects evoked by hCG $\beta$ are related to apoptosis suppression.
\end{abstract}

\section{Introduction}

Human chorionic gonadotropin (hCG), which together with lutropin ( $\mathrm{LH})$, thyrotropin (TSH) and follitropin (FSH) belong to one glycoprotein hormone family, is produced during pregnancy by trophoblast cells (1). The hormone controls a number

Correspondence to: Professor Anna Jankowska, Department of Cell Biology, Poznan University of Medical Sciences, Rokietnicka 5D, 60-806 Poznan, Poland

E-mail: ajanko@ump.edu.pl

${ }^{*}$ Contributed equally

Key words: human chorionic gonadotropin, apoptosis, $B C L 2, B A X$, BIRC5, LHCGR, ovarian cancer of processes, including embryo implantation, angiogenesis and development of the chorion (2).

The hormone is a glycoprotein composed of subunits $\alpha$ (hCG $\alpha$ ) and $\beta$ (hCG $\beta$ ). Expression of human chorionic gonadotropin and in particular its $\beta$ subunit $(\mathrm{hCG} \beta)$ has been also documented for $30-60 \%$ of tumors of different origin $(2,3)$. What is more, it has been shown that transcriptional activity of genes encoding hCG $\beta$ is higher in advanced cancers. Its presence in serum and urine of cancer patients and in tumor tissues in many cases is of prognostic significance as it correlates with a poor response to therapy and thus poor prognosis (4).

It is postulated that, similarly to the way chorionic gonadotropin promotes angiogenesis during placenta formation and modulates the mother's immune system towards immunotolerance of the fetus, the hormone can play analogous functions in oncogenesis. This action of CG is said to contribute to neovascularization as well as desensitization of the immunological system towards cancer cells $(5,6)$. Several publications also demonstrate that $\mathrm{CG}$ promotes tumor growth by exhibiting anti-apoptotic and/or proliferative effects $(7,8)$.

Despite numerous studies on the role of CG and its free subunits in carcinogenesis, the biological functions and mechanisms behind their action remain unknown.

One hypothesis, which explains hCG $\beta$ action in cancers, is based on the hormone's structural similarity to growth factors such as TGF $\beta$ (transforming growth factor $\beta$ ), PDGF $\beta$ (platelet-derived growth factor $\beta$ ) and NGF (nerve growth factor) characterized by the presence of a cysteine knot motif. It is suggested that due to this structural similarity, $\mathrm{CG}$, like the aforementioned growth factors, may affect cells by regulation of their proliferation (9).

Recent studies have shown that the interaction of the $\beta$ subunit of hCG with its receptor (LHCGR, luteinizing hormone/choriogonadotropin receptor) may lead to initiation of signaling cascades associated with extracellular signalregulated kinases (ERK) and protein kinase $\mathrm{B}(\mathrm{PKB} / \mathrm{Akt})$. The kinases are involved in cell cycle regulation, apoptosis and cancer pathogenesis $(6,10)$. Thus, $\mathrm{CG}$ might also be considered a tumor-growth promoter.

Results of in vitro studies obtained independently by Hamada et al and our group support this hypothesis showing that silencing of $C G B$ genes induces apoptosis $(11,12)$. We demonstrated that the inhibition of CGB expression caused apoptosis both in cells expressing the anti-CGB construct as 
well as in neighboring cells, lacking the construct. This neighboring cell effect could be explained by the overall decreased level of hCG $\beta$ in culture medium, a state which presumably allows more interactions between receptors and ligands essential for induction of apoptosis (12).

The in vitro results were verified on animal models. These experiments proved in turn that non-cancerous ovarian epithelial cells stably transfected with $C G B$ overexpressed BCL-X(L) (B-cell lymphoma-extra large) and were characterized by a lower rate of apoptosis as well as more intense proliferation with increased levels of cyclin E/D1 and Cdk2/4/6. In consequence, cell phenotype changes led to increased tumorigenesis of xenografts in athymic nude mice. Histopathological analysis of tumors arising from implanted xenografts overexpressing hCG $\beta$ demonstrated that the cells were poorly differentiated (13).

What is more it has been shown that expression of hCG $\beta$ correlates with a decreased apoptosis rate in human cervical carcinomas (14). This suggests that the presence of hCG, produced by tumors, protects cells from initiation of apoptosis, allowing tumor development and growth.

Thus, in the present study we attempted to verify the association between expression of $C G B$ and factors regulating apoptosis such as $B C L 2$ (B-cell lymphoma 2), $B A X$ (Bcl-2-like protein 4) and BIRC5 (survivin).

\section{Materials and methods}

Specimens of ovarian cancer tissue were obtained from 45 patients with ovarian cancer treated by surgery at the Department of Gynecologic Oncology, Poznan University of Medical Sciences. Histological subtypes and the grade of the carcinomas are presented in Table I.

The control group included samples of ovaries $(n=8)$ and fallopian tubes $(n=6)$ that lacked cancerous transformations as assessed by macroscopic and microscopic examination by a pathologist. Control samples were obtained from postmenopausal patients who underwent total hysterectomy with additional oophorectomy due to myomas.

Both ovarian cancer tissues and control tissues were maintained in RNAlater buffer (Sigma Life Sciences, St. Louis, $\mathrm{MO}, \mathrm{USA})$ at $-80^{\circ} \mathrm{C}$ prior to further processing.

The study was approved by the Ethics Review Board of Poznan University of Medical Sciences (resolution no. 748/08) and all patients participated after informed consent.

SKOV-3 and OVCAR-3 cell lines, established from ovarian carcinomas and used as an in vitro model of cancer, were obtained from the Global Bioresource Center, American Type Culture Collection (ATCC, Manassa, VA, USA; SKOV-3 ATCC ${ }^{\circledR}$ HTB-77 $^{\mathrm{TM}}$, OVCAR-3 ATCC ${ }^{\circledR}$ TB-161 ${ }^{\mathrm{TM}}$ ). The choice of cell lines was determined by the fact that SKOV-3 is characterized by the lack of LHCGR expression, while OVCAR-3 cells express the receptor (15). The presence and lack of LHCGR expression in the analyzed cells was also validated experimentally by quantitative polymerase chain reaction (qPCR) (data not shown). Cells were cultured and passaged under standard conditions.

Cell culture and transfection. Cells were seeded so as to at the time of transfection obtain the optimal 70-80\% confluence.
Table I. The histological subtype and grade of the studied ovarian carcinomas.

\begin{tabular}{lrccc}
\hline & \multicolumn{3}{c}{ Ovarian carcinoma subtype } \\
\cline { 2 - 5 } Tumor grade & Serous & Endometrial & Mucinous & $\begin{array}{c}\text { Clear } \\
\text { cell }\end{array}$ \\
\hline G1 & 0 & 0 & 0 & 0 \\
G2 & 10 & 5 & 2 & 2 \\
G3 & 22 & 3 & 0 & 0 \\
Not determined & 2 & 0 & 0 & 0 \\
Total & 34 & 8 & 2 & 2 \\
\hline
\end{tabular}

Transfection with the construct carrying the $C G B 5$ gene, chosen as one of the most transcriptionally active $C G B$ genes (17), utilizing TurboFect (Thermo Scientific, Rockford, IL, USA) was performed according to the manufacturer's protocol. The CGB5 construct was prepared as described previously (16).

Non-transfected cells were used as control. Additionally in order to estimate the transfection efficiency, an equal amount of reporter eGFP plasmid (Invitrogen, San Diego, CA, USA) was used to transfect the study cell lines.

Analyses were conducted $72 \mathrm{~h}$ after transfection. All experiments were performed in triplicates.

RNA isolation. Total RNA from the SKOV-3 and OVCAR-3 cells as well as from the analyzed tissues (100-300 mg) was isolated using TriPure Isolation reagent (Roche Diagnostics, Mannheim, Germany) according to the manufacturer's protocol. An air-dried pellet of RNA was suspended in UltraPure DNase/RNase-Free Distilled Water (Invitrogen, Carlsbad, CA USA). RNA was stored at $-80^{\circ} \mathrm{C}$ prior to subsequent steps.

cDNA synthesis. One microgram of RNA was used for each reverse transcription reaction with universal primer $\mathrm{p}(\mathrm{dT}) 10$ and Transcriptor Reverse Transcriptase (Roche Diagnostics), according to the delivered protocol.

$q P C R$. To assess the expression level of the analyzed genes, qPCR with sequence-specific primers, hydrolysis probes and the LightCycler ${ }^{\circledR}$ TaqMan ${ }^{\circledR}$ Master kit (Roche Diagnostics) was performed. Probes and primers used in the reactions are presented in Table II.

The reaction mix for the TaqMan reactions contained: $5 \mu \mathrm{l}$ of cDNA, 1X TaqMan Master Mix (Roche Diagnostics), $0.1 \mu \mathrm{M}$ hydrolysis probe (TaqMan) and $0.4 \mu \mathrm{M}$ of each primer. qPCR program consisted of initial denaturation at $95^{\circ} \mathrm{C}$ for 10 min followed by 453 -step cycles: $95^{\circ} \mathrm{C} / 10 \mathrm{sec}$ hold for denaturation, $60^{\circ} \mathrm{C} / 30 \mathrm{sec}$ hold for primers and probe hybridization and product extension, and $72^{\circ} \mathrm{C} / 1 \mathrm{sec}$ hold for data acquisition.

Relative expression of genes analyzed by TaqMan assays was normalized against HPRT expression (Human HPRT Gene Assay; Roche Diagnostics). All experiments were performed in triplicates using independently synthesized cDNA. 
Table II. Primers and hydrolysis probes used in qPCR assays.

\begin{tabular}{ll}
\hline Gene(s) & \multicolumn{1}{c}{ Starters and probes } \\
\hline CGB1-9 & 5'-TACTGCCCCACCATGACC-3' \\
& 5'-CACGGCGTAGGAGACCAC-3' \\
& \#71 (Roche Diagnostics UPL) \\
LHCGR & 5'-CACTCGACTATCATCTGCCTACCTCC-3' \\
& 5'-GAAAAGCATTTCCTGGTATGGTGG-3' \\
& 6FAM-CAGGCATCAGAAAGTTTCCAGATGTTACGA- \\
& BBQ \\
& (TIB MOLBIOL) \\
BAX & 5'-ATGTTTCTGACGGCAACTTC-3' \\
& 5'-ATCAGTTCCGGCACCTTG-3' \\
& \#57 (Roche Diagnostics UPL) \\
BCL2 & 5'-TACCTGAACCGGCACCTG-3' \\
& 5'-GCCGTACAGTTCCACAAAGG-3' \\
& \#75 (Roche Diagnostics UPL) \\
BIRC5 & 5'-TCTGCTTCAAGGAGCTGGA-3' \\
& 5'-AAAGTGCTGGTATTACAGGCGTA-3' \\
& \#36 (Roche Diagnostics UPL) \\
HPRT & Human HPRT Gene Assay cat no. 05046157001 \\
& (Roche Diagnostics UPL)
\end{tabular}

National Center for Biotechnology Information reference sequence: CGB1-NM_033377.1; CGB2-NM_033378.1; CGB3-NM_000737.3; CGB5NM_033043.1; CGB7-NM_033142.1; CGB8-NM_033183.2; LHCGRNM_000233.3; $B A X$-NM_001291428.1 (isoform 1), NM_138761.3 (isoform $\alpha$ ), NM_004324.3 (isoform $\beta$ ), NM_001291429.1 (isoform $\gamma$ ), NM_138763.3 (isoform $\delta$ ), NM_001291430.1 (isoform $\lambda$ ), NM_001291431.1 (isoform $\zeta$ ); $B C L 2$-NM_000633.2 (isoform $\alpha$ ), NM_000657.2 (isoform $\beta$ ); BIRC5- NM_001168.2 (isoform 1); NM_001012270.1 (isoform 2), NM_001012271.1 (isoform 3); HPRT-NM_0001942.2.

Statistical analysis. Data were analyzed using the Statistica 10 software package (StatSoft, Krakow, Poland) with Mann-Whitney U test in case of cell lines and Kruskal-Wallis test with Dunn's post hoc test in case of tissues. Differences were considered statistically significant if $\mathrm{p}$-value was $<0.05$. Associations between particular gene expression were tested using Spearman's rank correlation.

For graphical representation, qPCR raw data were transformed and presented as the logarithm to base ten.

\section{Results}

Overexpression of $C G B$ affects the expression of $B C L 2, B A X$ and BIRC5 in the ovarian cancer cell lines. In order to test the hypothesis that hCG $\beta$ synthesized by cancer cells protects them against the induction of apoptosis, ovarian cancer cell lines, OVCAR-3 and SKOV-3, were transfected with a construct carrying the $C G B 5$ gene, which is one of the most transcriptionally active $C G B$ genes (17).

Introduction of the construct into OVCAR-3 and SKOV-3 cells resulted on average in $80 \%$ efficiency of transfection and thus a profound overexpression of $C G \beta$ subunit mRNA in both cell lines. The relative level of $C G B 1-9$ transcripts in the transfected OVCAR-3 and SKOV-3 cells was on average $>3,000$ - and 15,000-fold higher than that in the control (non-transfected) cells, respectively. Differences for both
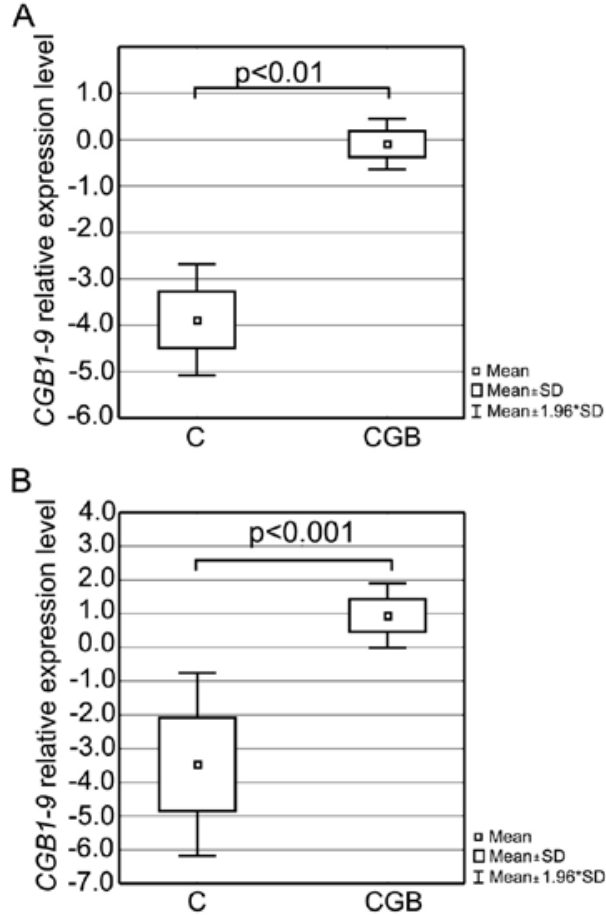

Figure 1. Relative expression levels of CGB1-9 genes in OVCAR-3 (A) and SKOV-3 (B) cells after transfection with the construct carrying CGB5. $\mathrm{C}$, control cells; CGB, transfected cells.

cell lines were statistically significant $(\mathrm{p}<0.001$ and $\mathrm{p}<0.001$, respectively, Fig. 1).

Analysis of $B C L 2$ and $B A X$ expression in the cell lines overexpressing $C G B 5$ showed that the mRNA level of these agents declined in the transfected cells (Fig. 2A and B and Fig. 3A and B). In the case of the transfected OVCAR-3 cells, expression of $B C L 2$ and $B A X$ was on average 2-fold lower than that in the control cells; differences were statistically significant ( $p=0.05$ and 0.05 , respectively). In the SKOV-3 cells, significant differences were noted for $B A X$ expression only $(\mathrm{p}<0.01)$, where in the control cells the gene expression was on average $>4$-fold lower.

No significant differences in the $B C L 2$ to $B A X$ expression ratio were observed between the control and transfected cell (Fig. 2C and Fig. 3C).

Similarly to $B C L 2$ and $B A X$, expression level of the $B I R C 5$ gene encoding survivin was noted to decline in the transfected cells (Fig. 2D and Fig. 3D). Statistically significant differences were noted between the control and transfected SKOV-3 cells $(\mathrm{p}<0.01)$; transfected cells were characterized by an average $>3$-fold lower BIRC5 expression than the control cells.

Since the physiological effect of $\mathrm{CG}$ is mediated via the receptor for luteinizing hormone and hCG, transfected cells were analyzed in terms of the receptor expression. In the case of OVCAR-3 cells, CGB5 overexpression was associated with an almost 2-fold decrease in the relative level of $L H C G R$ mRNA, but the difference was not statistically significant (Fig. 4). Increased expression of $C G B 5$ in the SKOV-3 cells, lacking LHCGR, did not influence the expression of the receptor; no LHCGR expression was noted (data not shown).

All analyses were made on the basis of three independent experiments and from each three independent experiments, cDNA was used for qPCR. 

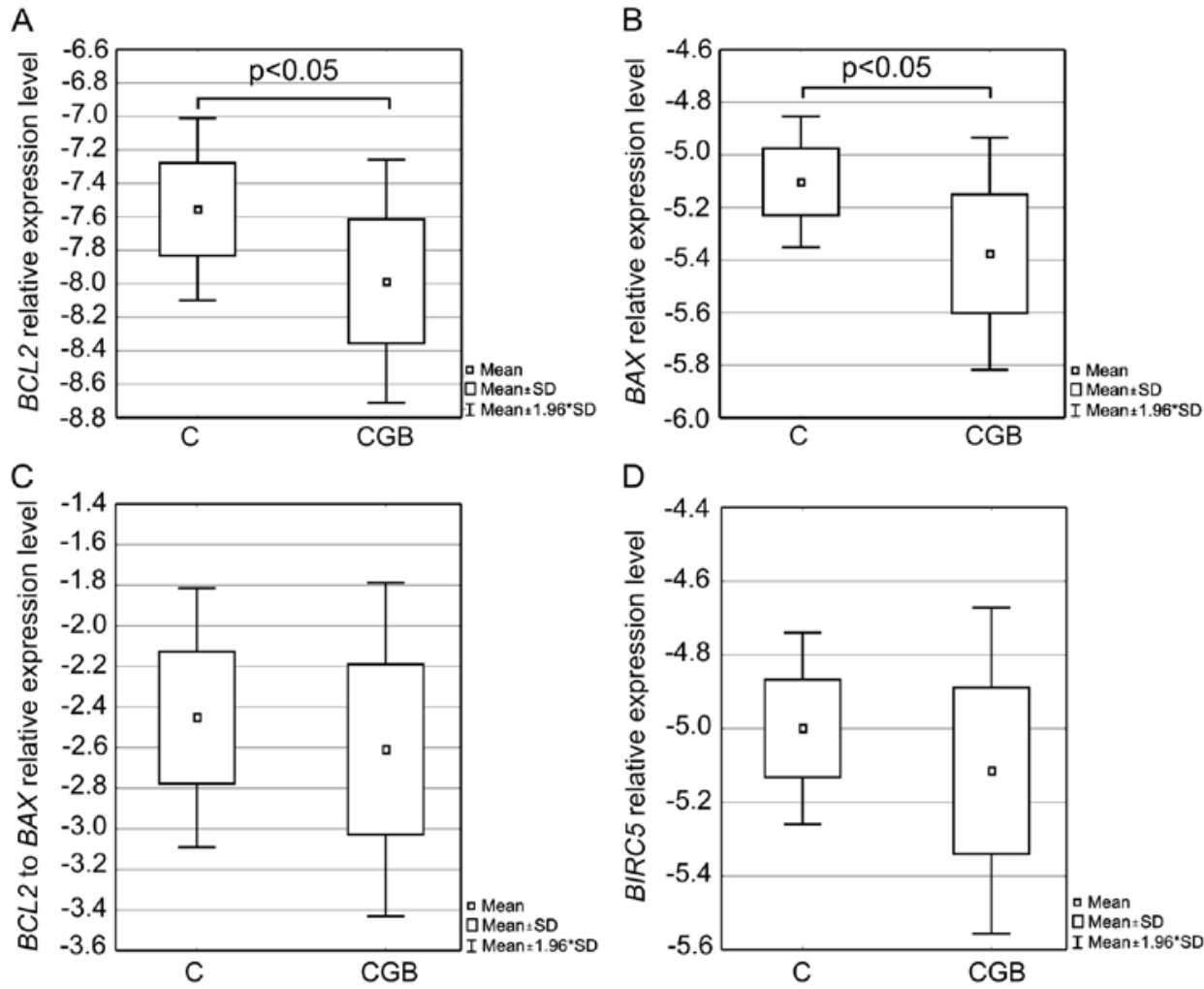

Figure 2. Effect of $C G B$ overexpression on the relative expression level of $B C L 2$ (A), $B A X(\mathrm{~B}), B I R C 5$ (D) and the $B C L 2 / B A X$ ratio (C) in OVCAR-3 cells. $\mathrm{C}$, control cells; CGB, transfected cells.

A
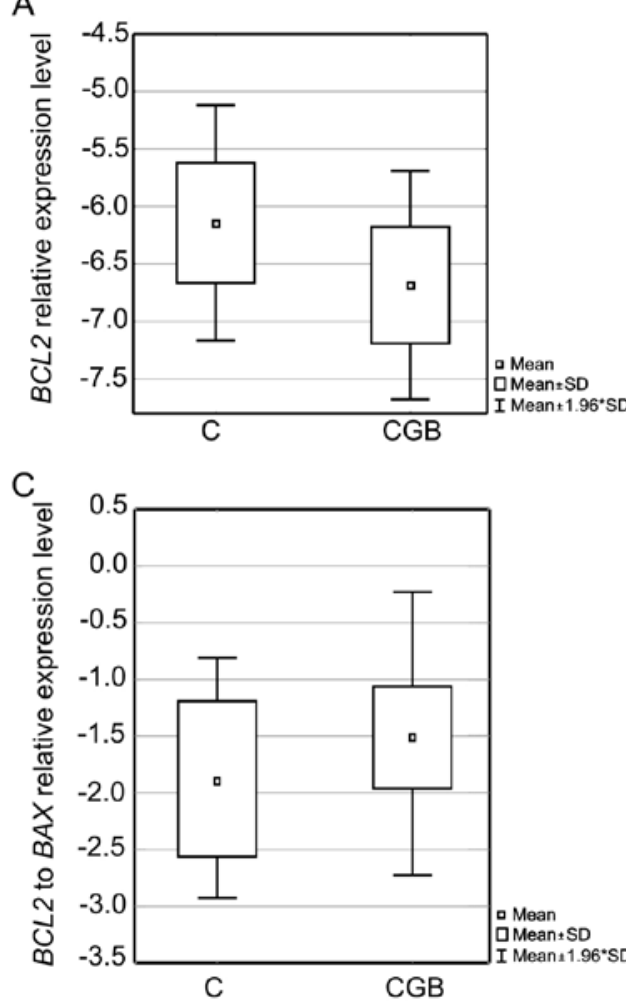

B

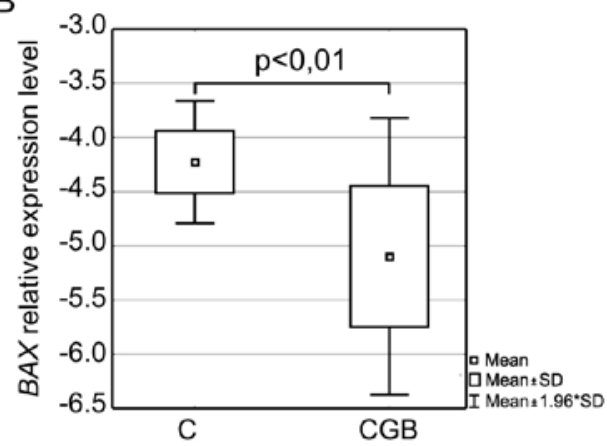

D

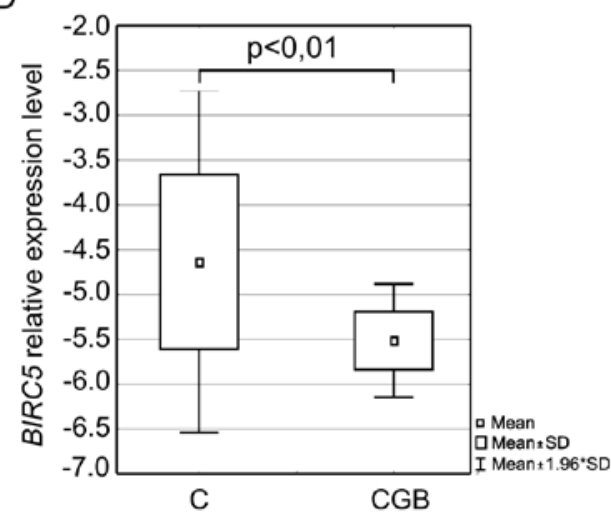

Figure 3. Effect of $C G B$ overexpression on the relative expression level of $B C L 2$ (A), $B A X$ (B), BIRC5 (D) and the $B C L 2 / B A X$ ratio (C) in SKOV-3 cells. C, control cells; CGB, transfected cells.

Ovarian cancer is characterized by altered expression of the BCL2, BAX and BIRC5 genes. The experiments conducted using RNA isolated from ovarian cancers, fallopian tubes and healthy ovaries showed that all analyzed tissues expressed 


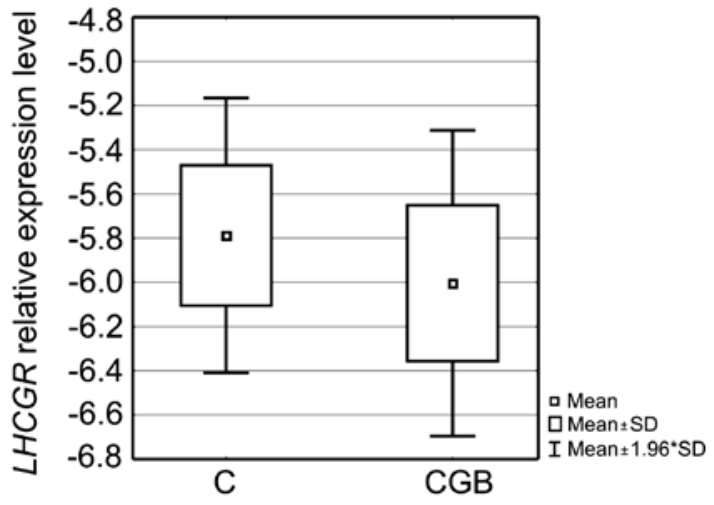

Figure 4. The effect of $C G B$ overexpression on the relative expression level of the LHCGR gene in OVCAR-3 cells. C, control cells; CGB, transfected cells.

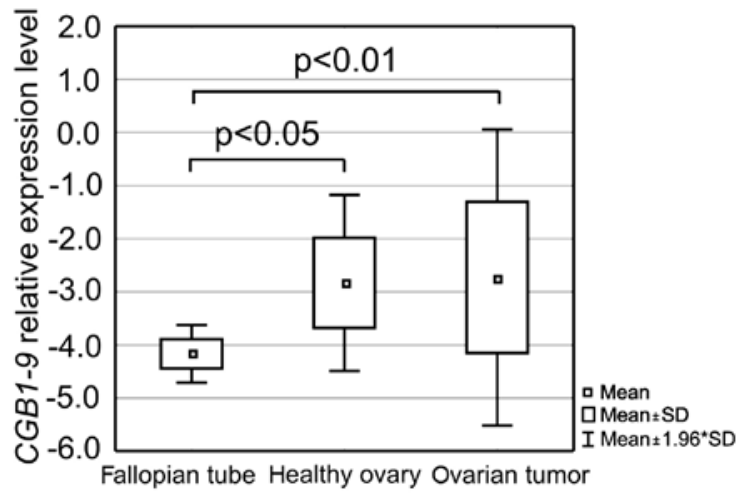

Figure 5. Relative expression level $(\log )$ of the CGB1-9 genes in the studied tissues: fallopian tubes, healthy ovaries and ovarian cancer.
$C G B 1-9$ and the level of gene expression differed between the groups (Fig. 5).

Fallopian tube tissues were characterized by lower $C G B 1-9$ expression than the other analyzed groups. On average it was $\sim 60,000$ - and 40,000-fold lower than the level in the healthy ovaries and ovarian cancer, respectively. Thus, statistically significant differences were observed between the group of fallopian tubes and healthy ovaries $(\mathrm{p}<0.05)$ as well as between fallopian tubes and ovarian cancers $(\mathrm{p}<0.01)$.

Assessment of the $B C L 2$ gene, encoding an anti-apoptotic factor, showed that ovarian cancers had a statistically significantly lower $B C L 2$ expression level than the fallopian tube $(p<0.001)$ and healthy ovary tissues $(p<0.01$, Fig. $6 \mathrm{~A})$ and that it was on average almost 30- and 10-fold lower, respectively. An opposite phenomenon was observed in case of the pro-apoptotic $B A X$ gene analysis. Evaluation of $B A X$ expression showed that the group of ovarian cancer tissues was characterized by distinctly higher amount of the gene transcripts than the fallopian tubes and healthy ovaries (Fig. 6B). On average it was almost 50 - and $>100$-fold higher, respectively, and the differences proved to be statistically significant $(\mathrm{p}<0.01$ and $\mathrm{p}<0.001$, respectively).

Consequently a decrease in the $B C L 2$ to $B A X$ expression ratio was reported (Fig. $6 \mathrm{C}$ ). Differences in the $B C L 2 / B A X$ between the ovarian cancer and fallopian tube tissues as well as the ovarian cancers and healthy ovaries were statistically significant $(\mathrm{p}<0.001$ and $\mathrm{p}<0.0001$, respectively).

The BIRC5 gene, coding for yet another factor regulating apoptosis, was shown to have on average a $>30$-fold higher expression level in case of the ovarian cancer than the level in the fallopian tube and healthy ovarian tissues. Differences in BIRC5 expression between the groups were also found to be statistically significant ( $<<0.01$ and $p<0.001$, respectively, Fig. 6D).

In all studied tissues, the expression level of the receptor for luteinizing hormone and human chorionic gonadotropin was also evaluated. The results confirmed that not all analyzed samples expressed $L H C G R$ at the RNA level. In 13 out of 45 ovarian cancer tissue samples, $L H C G R$ expression was found to be below the adopted qPCR assay's detection range. In these cases zero was replaced with a value $10 \%$ lower than the lowest $L H C G R$ expression level observed in the assay. This allowed performing full statistical analysis, which proved that again the ovarian cancer tissue expression profile was different from the profiles of the fallopian tube and healthy ovarian tissues. The ovarian cancer tissue group was discriminated by a lower $L H C G R$ expression level than the level in the other two analyzed groups. It was especially distinct between cancers and fallopian tubes as it was on average $>10$-fold lower. Differences in the gene expression between ovarian cancer and fallopian tube tissues as well as ovarian cancer and healthy ovarian tissues were shown to be statistically significant $(\mathrm{p}<0.001$ and $\mathrm{p}<0.05$, respectively, Fig. 7).

Spearman's rank order correlation analysis pointed to statistically significant relations within the three studied tissue groups. Among the cancer tissue cases, the relative level of LHCGR transcripts was negatively correlated with the expression level of BIRC5 encoding survivin ( $\mathrm{p}<0.05 ; \mathrm{R}=-0.39)$.

On the other hand, in the group of fallopian tube tissues, LHCGR expression level showed a very strong positive correlation with the number of $B C L 2$ transcripts ( $\mathrm{p}<0.05 ; \mathrm{R}=0.94)$.

The highest number of statistically significant correlations was however noted for the group of healthy ovaries. In these tissues, $C G B$ expression showed a very strong positive correlation with survivin expression $(\mathrm{p}<0.05 ; \mathrm{R}=0.80)$ as well as a strong negative correlation with transcriptional activity of $L H C G R(\mathrm{p}<0.05 ; \mathrm{R}=-0.74)$. In this group, $B A X$ expression was also correlated positively with the $B C L 2$ expression level ( $\mathrm{p}<0.05$; $\mathrm{R}=0.74$ ), while $L H C G R$ was correlated negatively with the survivin mRNA level $(\mathrm{p}<0.05 ; \mathrm{R}=-0.79)$.

All analyses were carried out based on the results obtained in qPCR with the use of three independent cDNAs synthesized for each RNA sample.

\section{Discussion}

Production of CG and its free $\beta$ subunit by tumors of different origins is a well-known phenomenon (4). However, the mechanism by which these interchangeable cancer promoters achieve their biological effects is not fully understood. The two most often pursued hypotheses concern direct influence on cancer cell proliferation or/and indirect cancer cell survival promotion by inhibiting apoptosis signals $(7,11,12,18)$.

The results of our previous studies as well as data published by Hamada's group demonstrated that silencing of the chorionic gonadotropin $\beta$ subunit leads to induction of 
A

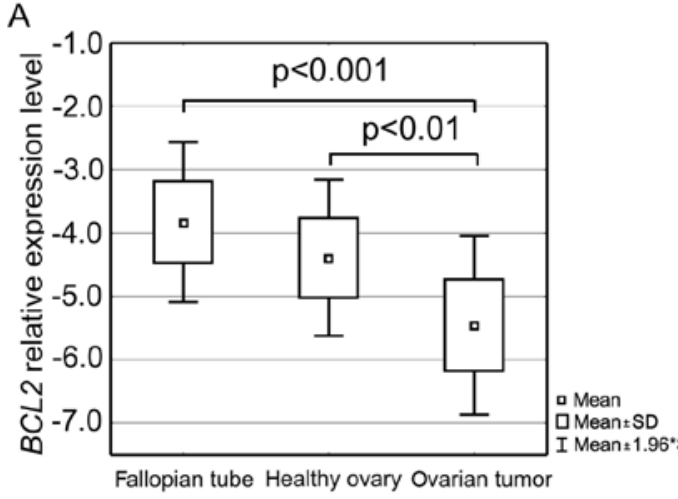

C

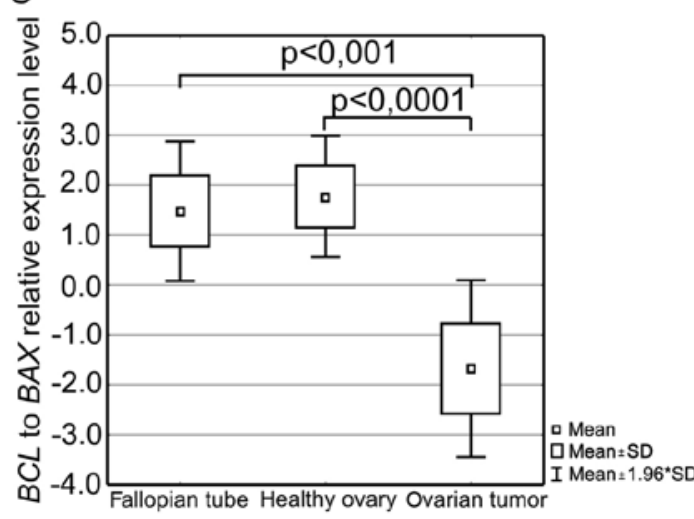

$\mathrm{B}$

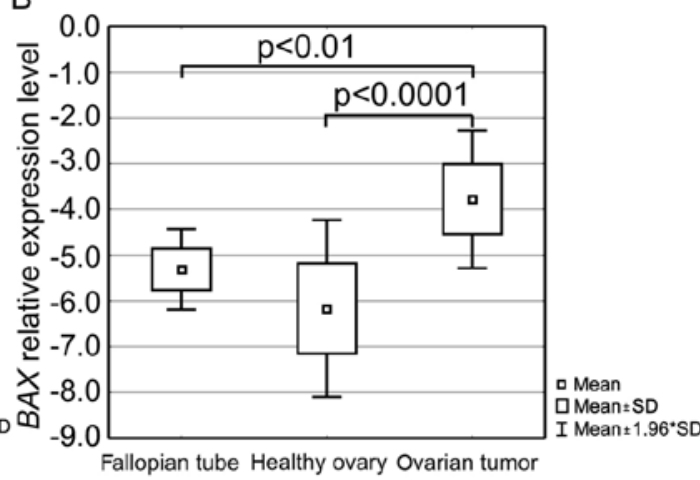

D

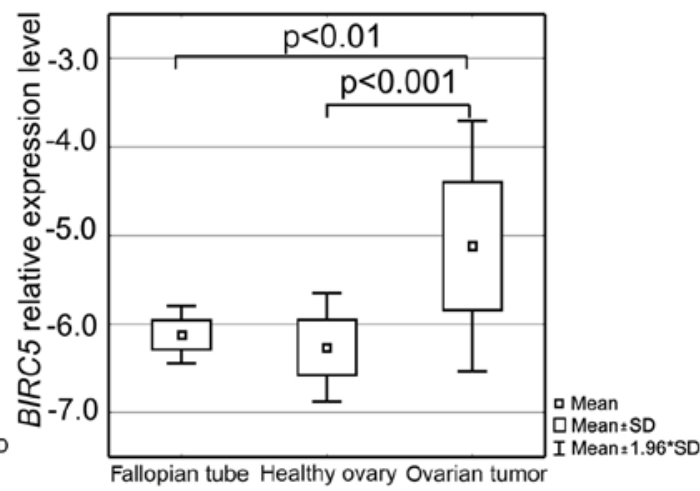

Figure 6. Relative expression level of $B C L 2$ (A), $B A X(\mathrm{~B}), B I R C 5$ (D) and the $B C L 2 / B A X$ ratio (C) in the studied tissues: fallopian tubes, healthy ovaries and ovarian cancer.

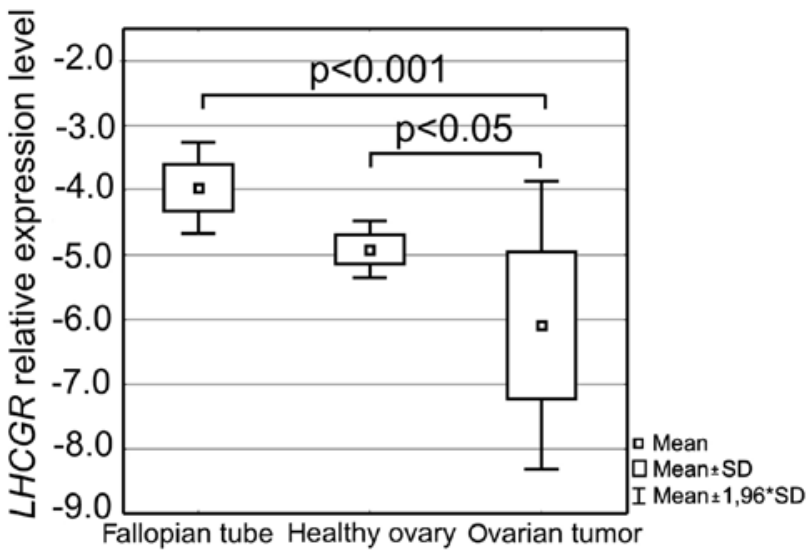

Figure 7. Relative expression level of the $L H C G R$ gene in the studied tissues: fallopian tubes, healthy ovaries and ovarian cancer.

programmed cell death in cancer cells cultured in vitro $(11,12)$. What is more it was shown that the expression of hCG $\beta$ was correlated with a decreased apoptosis rate in human cervical carcinoma samples analyzed by IHC (14). This suggests that $\mathrm{hCG}$ produced by tumors may protect cells from initiation of apoptosis, allowing tumor development and growth.

In the present study, the expression level of $B C L 2, B A X$, and BIRC5 genes involved in the apoptosis of ovarian cancers expressing CGB was analyzed. Selection of these factors was based on the fact that they are key players in the activation of caspases in the final stages of apoptosis. Moreover, their aberrant expression marks a variety of tumors (19).
In the first step of the study, the influence of hCG $\beta$ on the expression of $B C L 2, B A X$ and $B I R C 5$ in an in vitro model was examined. Introduction of the construct carrying the $C G B 5$ gene into OVCAR-3 and SKOV-3 cells resulted in a profound, statistically significant increase in $C G B$ expression in both cell lines.

Overexpression of the hormone's $\beta$ subunit caused a decrease in $B C L 2$ and $B A X$ transcript level in both transfected cell lines. However no significant differences were observed in the $B C L 2$ to $B A X$ expression ratio between the transfected and control cells.

Similarly to $B C L 2$ and $B A X$, the expression of the $B I R C 5$ gene encoding survivin was also noted to decline in the transfected cells compared to the control (non-transfected) cells.

The same analyses were conducted on RNA isolated from the ovarian cancer tissues.

Ovarian cancer can be very heterogeneous at both the histological and genetic level. It was suggested recently that the source of both low- and high-grade serous carcinoma is the fallopian tube epithelium (benign or malignant) implanted on the ovary, rather than the ovarian surface epithelium itself, as previously believed (20). In the view of this new model of ovarian tumorigenesis, the control groups of our study comprised healthy ovaries and fallopian tubes.

All studied tissues, both cancer and healthy, were characterized by the presence of $C G B$ transcripts. However, the relative expression level of the hormone's subunit varied. Fallopian tube tissues were distinguished by a lower CGB1-9 expression level than that in the other analyzed tissues. Statistically significant differences were observed between the group of 
fallopian tubes and healthy ovaries as well as the fallopian tubes and ovarian cancers. Such diversity in $C G B$ expression in ovarian cancer was previously shown (21).

Analysis of apoptotic agents in ovarian cancers expressing $C G B$ demonstrated a significantly lower $B C L 2$ mRNA level in cancer than in the fallopian tubes and healthy ovaries. In contrast, a higher level of $B A X$ gene transcripts was found in the ovarian cancer tissues compared to the control groups.

Changes in the expression of BCL2 and BAX are essential for the regulation of cell cycle and apoptosis. Overexpression of BCL2 protects normal cells from apoptosis (22), whereas excess of BAX can lead to cell death induction (23). Thus, the level of both factors in ovarian cancer, regulated by hCG, may affect tumor cell growth and survival. This scenario seems possible since CG controlling the apoptosis of the corpus luteum through BCL2 and BAX was previously documented (24). On the other hand, these factors expressed in tumors may act differently than in normal tissues. In cancer, a high level of BCL2 protein is known to correlate with tumor differentiation and better prognosis, while overexpression of BAX with a worse prognosis. In ovarian cancer patients, BAX, but not BCL2 expression, correlates with significantly reduced survival compared to individuals characterized by the expression of both factors (25). Thus, the results of the present study, demonstrating increased transcriptional activity of the $B A X$ gene in ovarian cancer tissues, are in agreement with previously published data. What is more, they suggest that the difference in both $B C L 2$ and $B A X$ gene expression may be responsible for more malignant phenotype of cancer cells overexpressing CGB.

Numerous studies have shown that the quantitative ratio of BCL2 and BAX expression (BCL2/BAX) and their interactions are a key element of the cell fate (survival or death) $(26,27)$. $\mathrm{BCL} 2 / \mathrm{BAX}$ is a characteristic feature of a given cell type. A decline in BCL2/BAX observed in advanced stages of ovarian cancer is associated with the degree of tumor differentiation but appears to have no correlation with its proliferation rate (26).

Taking this into consideration, we estimated the BCL2/BAX ratio for all studied tissue groups. The highest value was noted for healthy tissues, fallopian tubes and ovaries, and the lowest characterized ovarian cancer tissue. Thus, these results and the fact that tumors expressing hCG $\beta$ are often metastatic and have worse prognosis may suggest that the $\beta$ subunit of hCG protects tumor cells from apoptosis and allows development of a malignant phenotype through modulation of $B C L 2$ and $B A X$ expression.

Aberrant apoptosis of ovarian cancer cells was additionally confirmed by the assessment of BIRC5 expression. The BIRC5 gene encodes survivin, a member of the IAP family proteins which play a role in oncogenesis via their effective suppression of apoptosis (27). Even though a direct link between $C G B$ and BIRC5 expression has not been shown, stimulation of survivin synthesis by CG in granulosa cells of the ovary was previously documented (28). This implies that survivin-mediated suppression of apoptosis in ovarian cancer is regulated by endogenously synthesized hCG $\beta$. The obtained results showed that the highest expression level of BIRC5 characterized cancer tissues. They are also in agreement with current research on the role of survivin in tumorigenesis, showing that a high level of survivin in tumors, similarly to CGB, is often associated with an invasive phenotype and resistance to chemotherapy and radiotherapy and consequently with poor prognosis (27).

In both cell lines $C G B$ overexpression led to a decrease in all analyzed apoptosis-related factors, while in the ovarian cancer tissues $C G B$ expression was associated with an increase in $B A X$ and BIRC5 expression. The reason why the results of our in vivo studies did not completely match the results of the in vitro study may be related to the fact that the in vitro model did not completely reflect the complexity of the tissue. Therefore, it must be taken into consideration that the final effect of hCG $\beta$ action in tissues depends on the interaction between different cell types and responses induced by the hormone.

CG acts via the receptor for luteinizing hormone and human chorionic gonadotropin, thus the expression level of $L H C G R$ in the studied cell lines and tissues was evaluated. SKOV-3 cells lack LHCGR, and overexpression of $C G B$ did not influence $L H C G R$ transcriptional activity. On the other hand, in the transfected OVCAR-3 cells, a decrease in the amount of $L H C G R$ mRNA was observed, however differences were not statistically significant.

These results were confirmed also by the analysis performed on RNA isolated from cancer tissues. In accordance with other studies showing that not all tumors with confirmed hCG $\beta$ expression were characterized by the presence of LHCGR (12), only part of the analyzed samples expressed $L H C G R$. In fact in 13 out of 45 ovarian cancer cases, LHCGR was not detected. What is more, ovarian cancers were discriminated by a significantly lower $L H C G R$ expression level than the one noted for healthy ovarian and fallopian tube tissues.

Thus, both in vitro and in vivo studies suggested that $C G B$ expression in cancer cells regulates $L H C G R$ expression. Negative LHCGR regulation under CG and hCG $\beta$ influence has been previously reported at both the mRNA and protein levels $(17,29)$. Our data together with the fact that hCG $\beta$ affects SKOV-3 cells lacking the receptor, suggests that the effect of the hormone on cancer cells is LHCGR-independent.

In the present study, the analyzed tumor tissues consisted of ovarian cancers, most of which, according to the newest classification, belonged to type II tumors (present in advanced stage and comprising high-grade serous, high-grade endometrioid, malignant mixed mesodermal tumors, carcinosarcomas, and undifferentiated carcinomas) (19). Since the analyzed cancer tissues were a rather homogenous group, no attempt was made to correlate the obtained results with clinical data.

The correlations we found linked $C G B$ positively with $B I R C 5$ and negatively with $L H C G R$ expression in healthy ovaries. Within this tissue group, strong positive correlation between $B A X$ and $B C L 2$ expression was also found. Surprisingly a very strong positive correlation between $L H C G R$ and $B C L 2$ in the fallopian tube group was noted while in cancer $L H C G R$ expression was negatively related with BIRC5. The meaning of these correlations is not clear.

In conclusion, even though the exact molecular mechanism of hCG $\beta$ action in cancer cells still needs to be established, the results of the present study together with the fact that tumors expressing the free $\beta$ subunit of hCG are characterized by a more malignant phenotype and worse prognosis, suggest that the biological effect of hCG $\beta$ is related to suppression of 
apoptosis. Protection of tumor cells from programmed cell death induction may be achieved by expression modulation of genes regulating apoptosis such as $B C L 2$, and $B A X$ and $B I R C 5$.

\section{Acknowledgements}

The present study was supported by the Polish National Science Centre Awards (NN 407459138 to A.S. and NN 407275439 to A.J.).

\section{References}

1. Pierce JG and Parsons TF: Glycoprotein hormones: Structure and function. Annu Rev Biochem 50: 465-495, 1981.

2. Cole LA: hCG, five independent molecules. Clin Chim Acta 413: 48-65, 2012.

3. Iles RK and Butler SA: Gonadotropins and gonadotropin receptors - evolutional genetics, signalling mechanisms, extra gonadal function and roles in oncogenesis. Mol Cell Endocrinol 329: 1-2, 2010.

4. Iles RK, Delves PJ and Butler SA: Does hCG or hCG $\beta$ play a role in cancer cell biology? Mol Cell Endocrinol 329: 62-70, 2010.

5. Tsampalas M, Gridelet V, Berndt S, Foidart JM, Geenen V and Perrier d'Hauterive S: Human chorionic gonadotropin: A hormone with immunological and angiogenic properties. J Reprod Immunol 85: 93-98, 2010.

6. Zygmunt M, Herr F, Keller-Schoenwetter S, Kunzi-Rapp K, Münstedt K, Rao CV, Lang U and Preissner KT: Characterization of human chorionic gonadotropin as a novel angiogenic factor. J Clin Endocrinol Metab 87: 5290-5296, 2002.

7. Burczynska BB, Kobrouly L, Butler SA, Naase M and Iles RK: Novel insights into the expression of CGB1 and 2 genes by epithelial cancer cell lines secreting ectopic free hCG $\beta$. Anticancer Res 34: 2239-2248, 2014.

8. Lee CL, Chiu PC, Hautala L, Salo T, Yeung WS, Stenman UH and Koistinen H: Human chorionic gonadotropin and its free $\beta$-subunit stimulate trophoblast invasion independent of $\mathrm{LH} / \mathrm{hCG}$ receptor. Mol Cell Endocrinol 375: 43-52, 2013.

9. Butler SA and Iles RK: The free monomeric beta subunit of human chorionic gonadotrophin (hCG beta) and the recently identified homodimeric beta-beta subunit (hCG beta beta) both have autocrine growth effects. Tumour Biol 25: 18-23, 2004.

10. Prast J, Saleh L, Husslein H, Sonderegger S, Helmer H and Knöfler M: Human chorionic gonadotropin stimulates trophoblast invasion through extracellularly regulated kinase and AKT signaling. Endocrinology 149: 979-987, 2008.

11. Hamada AL, Nakabayashi K, Sato A, Kiyoshi K, Takamatsu Y, Laoag-Fernandez JB, Ohara N and Maruo T: Transfection of antisense chorionic gonadotropin $\beta$ gene into choriocarcinoma cells suppresses the cell proliferation and induces apoptosis. J Clin Endocrinol Metab 90: 4873-4879, 2005.

12. Jankowska A, Gunderson SI, Andrusiewicz M, Burczynska B, Szczerba A, Jarmolowski A, Nowak-Markwitz E and Warchol JB Reduction of human chorionic gonadotropin beta subunit expression by modified U1 snRNA caused apoptosis in cervical cancer cells. Mol Cancer 7: 26-31, 2008.

13. Guo X, Liu G, Schauer IG, Yang G, Mercado-Uribe I, Yang F, Zhang S, He Y and Liu J: Overexpression of the $\beta$ subunit of human chorionic gonadotropin promotes the transformation of human ovarian epithelial cells and ovarian tumorigenesis. Am J Pathol 179: 1385-1393, 2011.
14. Li D, Wen X, Ghali L, Al-Shalabi FM, Docherty SM, Purkis P and Iles RK: hCG beta expression by cervical squamous carcinoma - in vivo histological association with tumour invasion and apoptosis. Histopathology 53: 147-155, 2008.

15. Mandai M, Konishi I, Kuroda H and Fujii S: LH/hCG action and development of ovarian cancer - a short review on biological and clinical/epidemiological aspects. Mol Cell Endocrinol 269: 61-64, 2007.

16. Głodek A and Jankowska A: CGB activates ERK and AKT kinases in cancer cells via LHCGR-independent mechanism. Tumour Biol 35: 5467-5479, 2014.

17. Hotakainen K, Lintula S, Jarvinen R, Paju A, Stenman J, Rintala E and Stenman UH: Overexpression of human chorionic gonadotropin beta genes 3,5 and 8 in tumor tissue and urinary cells of bladder cancer patients. Tumour Biol 28: 52-56, 2007.

18. Iles RK: Ectopic hCGbeta expression by epithelial cancer: Malignant behaviour, metastasis and inhibition of tumor cell apoptosis. Mol Cell Endocrinol 260-262: 264-270, 2007.

19. Philchenkov A: Caspases: Potential targets for regulating cell death. J Cell Mol Med 8: 432-444, 2004.

20. Kurman RJ: Origin and molecular pathogenesis of ovarian high-grade serous carcinoma. Ann Oncol 24 (Suppl 10): x16-21, 2013.

21. Kubiczak M, Walkowiak GP, Nowak-Markwitz E and Jankowska A: Human chorionic gonadotropin beta subunit genes CGB1 and CGB2 are transcriptionally active in ovarian cancer. Int J Mol Sci 14: 12650-12660, 2013.

22. Yang J, Liu X, Bhalla K, Kim CN, Ibrado AM, Cai J, Peng TI, Jones DP and Wang X: Prevention of apoptosis by Bcl-2: Release of cytochrome c from mitochondria blocked. Science 275: 1129-1132, 1997.

23. Yin C, Knudson CM, Korsmeyer SJ and Van Dyke T: Bax suppresses tumorigenesis and stimulates apoptosis in vivo. Nature 385: 637-640, 1997.

24. Sugino N, Suzuki T, Kashida S, Karube A, Takiguchi S and Kato $\mathrm{H}$ : Expression of Bcl-2 and Bax in the human corpus luteum during the menstrual cycle and in early pregnancy: Regulation by human chorionic gonadotropin. J Clin Endocrinol Metab 85: 4379-4386, 2000.

25. Basu A and Haldar S: The relationship between BcI2, Bax and p53: Consequences for cell cycle progression and cell death. Mol Hum Reprod 4: 1099-1109, 1998.

26. Yoon $\mathrm{O}$ and Roh J: Downregulation of KLF4 and the Bcl-2/Bax ratio in advanced epithelial ovarian cancer. Oncol Lett 4: 1033-1036, 2012.

27. Waligórska-Stachura J, Jankowska A, Waśko R, Liebert W, Biczysko M, Czarnywojtek A, Baszko-Błaszyk D, Shimek V and Ruchała M: Survivin - prognostic tumor biomarker in human neoplasms - review. Ginekol Pol 83: 537-540, 2012.

28. Kumazawa Y, Kawamura K, Sato T, Sato N, Konishi Y, Shimizu Y, Fukuda J, Kodama H and Tanaka T: HCG up-regulates survivin mRNA in human granulosa cells. Mol Hum Reprod 11: 161-166, 2005.

29. Hoffman YM, Peegel H, Sprock MJ, Zhang QY and Menon KM: Evidence that human chorionic gonadotropin/luteinizing hormone receptor down-regulation involves decreased levels of receptor messenger ribonucleic acid. Endocrinology 128: 388-393, 1991. 